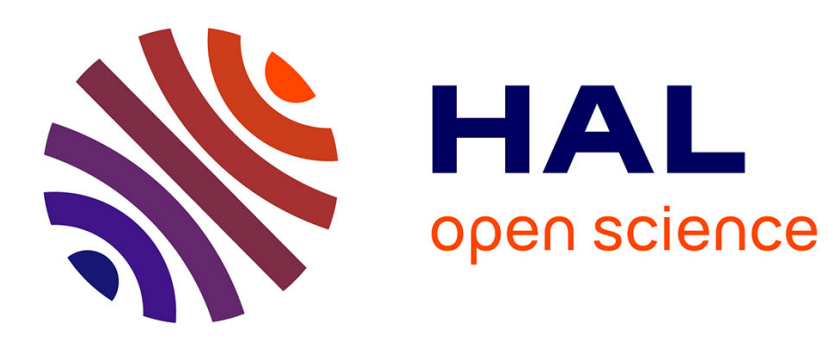

\title{
Electronic susceptibility of small particles of silver
}

R. Monot, J.-L. Millet

\section{To cite this version:}

R. Monot, J.-L. Millet. Electronic susceptibility of small particles of silver. Journal de Physique Lettres, 1976, 37 (3), pp.45-48. 10.1051/jphyslet:0197600370304500 . jpa-00231232

\section{HAL Id: jpa-00231232 https://hal.science/jpa-00231232}

Submitted on 1 Jan 1976

HAL is a multi-disciplinary open access archive for the deposit and dissemination of scientific research documents, whether they are published or not. The documents may come from teaching and research institutions in France or abroad, or from public or private research centers.
L'archive ouverte pluridisciplinaire HAL, est destinée au dépôt et à la diffusion de documents scientifiques de niveau recherche, publiés ou non, émanant des établissements d'enseignement et de recherche français ou étrangers, des laboratoires publics ou privés. 


\title{
ELECTRONIC SUSCEPTIBILITY OF SMALL PARTICLES OF SILVER
}

\author{
R. MONOT (*) and J.-L. MILLET \\ Laboratoire de physique expérimentale, E.P.F.-Lausanne, Switzerland \\ (Reçu le 27 juin 1975, révisé le 13 octobre 1975 et le 5 décembre 1975, accepté le 18 décembre 1975)
}

\begin{abstract}
Résumé. - Une étude entre $8 \mathrm{~K}$ et $190 \mathrm{~K}$ de petites particules d'argent en matrice de benzène a été faite par R.P.E. Pour des tailles de particules suffisamment petites pour présenter l'effet de taille quantique (diamètre $\sim 10 \AA$ ), une susceptibilité de type Curie a été mise en évidence. Ce fait est en accord avec les prévisions théoriques et l'écart moyen des niveaux d'énergie au voisinage du niveau de Fermi a pu être estimé.
\end{abstract}

\begin{abstract}
An E.P.R. study of small particles of silver in a benzene matrix has been performed between $8 \mathrm{~K}$ and $190 \mathrm{~K}$. For particle sizes sufficiently small to exhibit the quantum size-effect (diameters $\sim 10 \AA$ ) we show that the susceptibility obeys a Curie-like law. This result is in agreement with theoretical predictions, and an estimate of the mean level spacing near the Fermi level has been deduced.
\end{abstract}

Previously [1], it has been predicted that the electronic properties of sufficiently small metal particles will differ significantly from those of the bulk material. This difference is attributed to the so-called quantum size effect in which a localization of the electronic wavefunction due to the small size of the particle produces a splitting of the electronic energy levels. One of the most interesting predictions associated with this model is that, when the energy difference $\delta$ between two adjacent energy levels at the Fermi level is comparable to $k T$, the magnetic susceptibility is strongly modified. In particular, for particles with an odd number of electrons, Kubo has predicted that for $k T \ll \delta$, the electronic susceptibility will be of the Curie type rather than the temperature independent Pauli type which is characteristic of the bulk metal.

The purpose of this letter is to present the results of measurements of the variation of the susceptibility of silver particles as a function of temperature and to compare this variation with the existing theoretical predictions. The measurements reported here were performed at temperatures between 8 and $190 \mathrm{~K}$ using silver particles which were trapped in a benzene matrix and which had an average diameter of $10 \AA$. The susceptibility was deduced from the silver conduction electron spin resonance (C.E.S.R.) absorption by calculating the area under the imaginary part of the dynamic susceptibility measured as a

$\left(^{*}\right)$ Present address : Physics Department, University of California, Berkeley, Ca. 94720 U.S.A. function of magnetic field strength. The present results are related to a previous publication in which the observation of C.E.S.R. in small silver crystals was reported [2]. The line width of the observed C.E.S.R. signals for the small silver particles is much narrower than that found for the bulk metal, and this difference can be accounted for by the decrease in the relaxation rate as a result of the quantum size effect [3].

The thermodynamic properties of an ensemble of small metallic crystals are of course directly related to their electronic energy level distributions. If we assume that the shape of a small particle is a perfect sphere, then some of the energy levels will be degenerate. Kubo [1] has proposed that the irregularities in the shape of the particles will lift the degeneracy in the electronic energy levels and thus, in a hypothetical set of particles, each having $N$ atoms, every single small crystal will have its own energy spectrum, which is determined by specific boundary conditions. Kubo also assumed that the levels are randomly distributed with successive energy levels spacings given by a Poisson distribution. Gorkov and Eliashberg [4] have shown that this approach does not take into account the repulsion of the energy levels and they introduced, in the case of weak magnetic fields and low spin orbit coupling, an orthogonal ensemble and, in the case of strong spin orbit coupling, a symplectic ensemble (for a discussion see ref. [4]). Denton et al. [5] have calculated the free energy per particle for these different ensembles. Their results for the electronic susceptibility which were calculated assuming that the 
number of electrons per particle remains constant (canonical statistical distribution) are plotted in figure 1 . In the case of particles with an odd number of electrons the susceptibility at low temperature follows closely a Curie law and it is not strongly dependent on the statistical ensemble chosen. Therefore from a measurement of the odd particle susceptibility it is accordingly very difficult to distinguish between the different ensembles. For the even particles susceptibility the Poisson distribution gives a finite susceptibility at zero temperature whereas the orthogonal and symplectic ensembles both give a susceptibility equal to zero.

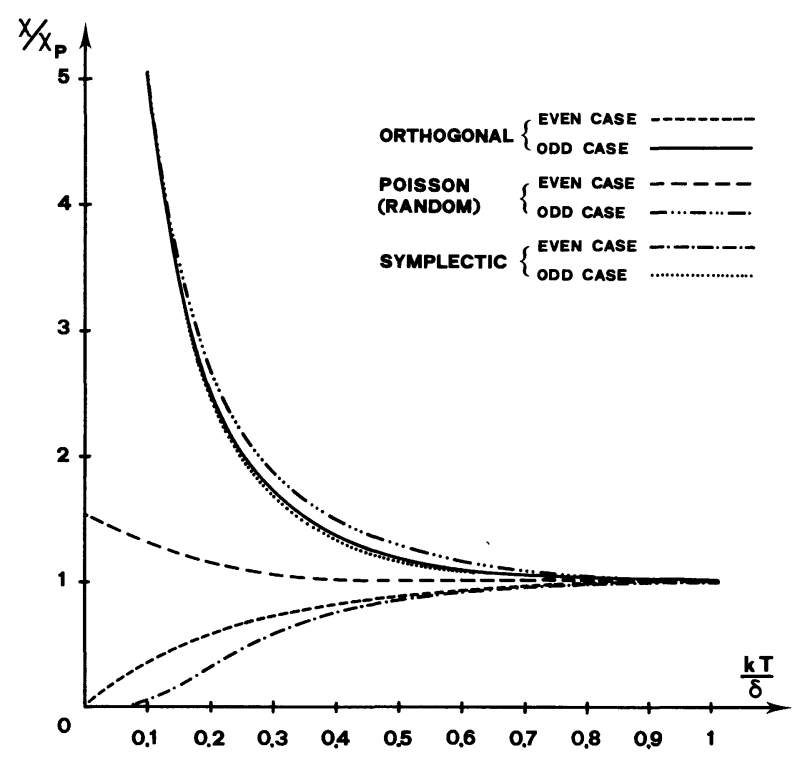

FIG. 1. - Spin susceptibility resulting from an average over the level distributions, normalized with respect to the Pauli value.

Experimental evidence for the differing susceptibilities of particles having an odd or an even number of atoms was found first by Taupin [6] from N.M.R. Knight shift measurements on small Li particles in LiF. Similar measurements performed more recently for small $\mathrm{Al}[7]$ and $\mathrm{Cu}[8]$ particles have shown that the particles with an even number of atoms tend to exhibit a zero Knight shift and hence a zero spin susceptibility. This proves that the distribution of energy levels deduced from the symplectic or orthogonal ensemble is more realistic than the random distribution. Though good measurements of the spin susceptibility have been deduced from the Knight shift in the case of even particles, the size distribution of the particles in the samples has prevented the extraction of precise information about the susceptibility of odd particles.

The susceptibility deduced from the temperature dependence of the E.S.R. absorption is obtained from samples with a size distribution. If we make the reasonable assumption that the number of particles with an odd number of conduction electrons is equal to that with an even number, the low temperature susceptibility is chiefly that of the odd particles. As already pointed out the odd particles susceptibility for the different distributions are close to each other and we cannot expect to determine which one applies to the case of silver.

The samples of small particles used in this experiment were prepared by simultaneously evaporating in a vacuum better than $1.5 \times 10^{-6}$ torr both silver and an inert matrix $\left(\mathrm{C}_{6} \mathrm{H}_{6}\right)$ onto a cold surface $(77 \mathrm{~K})$. The reported measurements were performed after annealing the samples at $195 \mathrm{~K}$. Before the annealing process the electron paramagnetic resonance signal was composed of four narrow hyperfine lines due to ${ }^{107} \mathrm{Ag}$ and ${ }^{109} \mathrm{Ag}$ atoms trapped in the matrix and a weak signal with an apparent $g$ value approximately equal to 2.007 [9]. During the annealing process the atomic silver hyperfine lines gradually disappear and the weak signal grows. We attribute this behaviour to silver atoms and clusters of atoms diffusing in the matrix to form small particles which are responsible for the signal whose $g$-value is approximately 2.007. If the sample temperature is raised above $195 \mathrm{~K}$ the central signal also disappears. We show in figure 2 the size distribution of the small particles, which was obtained with a transmission

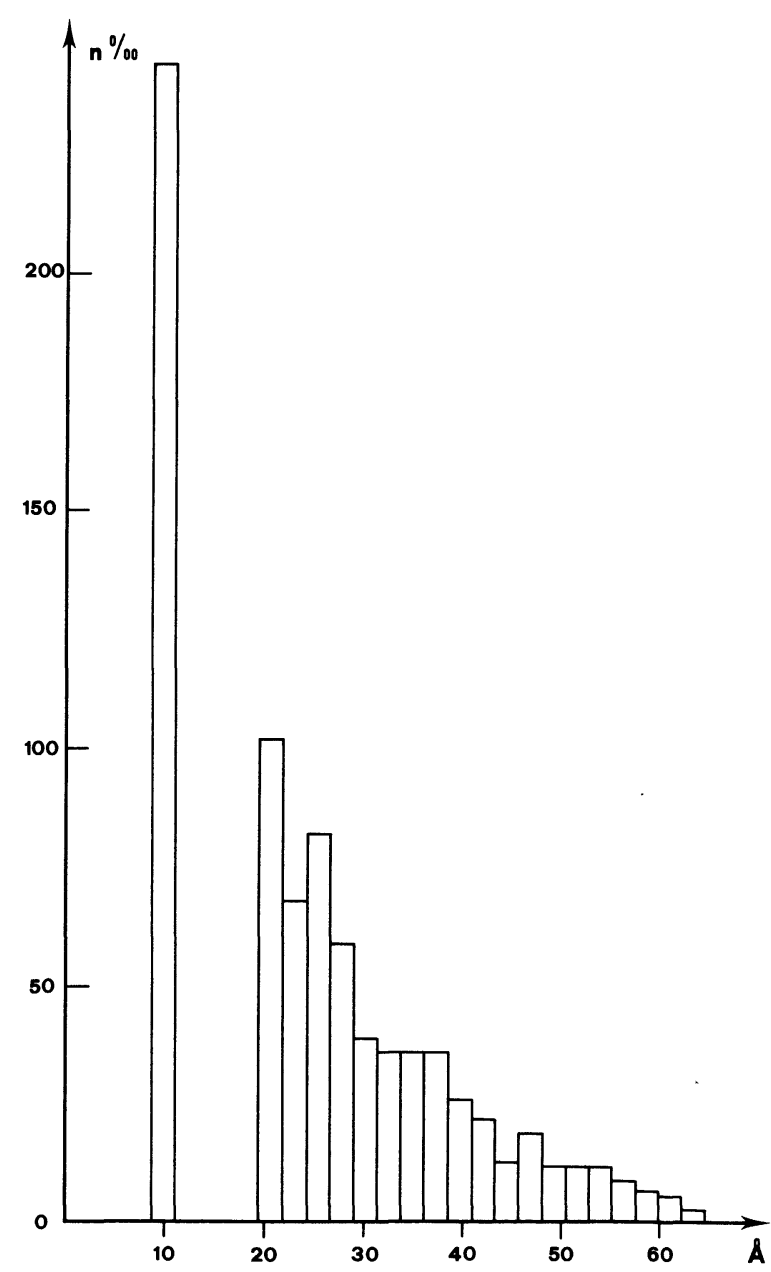

FIG. 2. - Size distribution of small Ag particles. 
electron microscope Philips EM 300 and a particle size analyser Quantimet.

It is well known that the molecular beam technique employed here can produce spurious electron paramagnetic resonance signals due to free radicals which are created during the deposition process. In order to check our results we have reproduced the experimental conditions used to prepare the small particles without silver in the heating basket. No electron paramagnetic resonance signal appeared either before or after the annealing process. We have also trapped silver particles in $\mathrm{C}_{18} \mathrm{H}_{38}$ or $\mathrm{CO}_{2}$ matrices, the atomic signals were less intense but after annealing the central signal was similar to that observed in $\mathrm{C}_{6} \mathrm{H}_{6}$. Using the same experimental set up we have evaporated lithium [10] and aluminium [11] metals. In the case of lithium trapped in a $\mathrm{CO}_{2}$ matrix the signal observed before annealing is only due to atomic hyperfine lines. After annealing at $160 \mathrm{~K}$ these lines disappear and a signal centered at $g$ equal 2 grows. The intensity of both the atomic signals and the central signal, that we attribute to small lithium particles nucleated during the annealing process, are in agreement with the amount of lithium in the matrix and the assumption of one paramagnetic center per small particle with an odd number of atoms. We thus believe that the above mentioned experimental results give strong support to our identification of the signal at $g$ around 2.007 as the signal of small silver particles.

The C.E.S.R. measurements were performed with a Varian X-band spectrometer. A flux of cold helium gas blown on the sample permitted a progressive variation of the temperature from $8 \mathrm{~K}$ up to $190 \mathrm{~K}$. The temperature was measured with an $\mathrm{Au}-\mathrm{Fe}$, $\mathrm{Ni}-\mathrm{Cr}$ thermocouple with an absolute precision of $\pm 2 \mathrm{~K}$. After completion of the whole series of measurement, a return of the sample to low temperature indicated that the size of the small particles had not changed during the process.

The susceptibility $\chi$ was measured through integration of the signal and direct comparison with the area under a similary integrated signal of a reference pitch. The experimental values of $\chi / \chi_{\mathrm{P}}$ where $\chi_{\mathrm{P}}$ is the Pauli susceptibility are given in figure 3 as a function of $k T / \delta$ where the mean energy spacing at the Fermi level is defined by $\delta=4 \varepsilon_{\mathrm{F}} / N$ [1]. In this formula $N$ is the number of atoms per small particle if we assume that all particles have the same number of $N$ atoms and $\varepsilon_{\mathrm{F}}$ is the Fermi energy. It should be pointed out that the Curie-like behaviour of $\chi$ does not depend on the assumptions made in the analysis of the C.E.S.R. spectra, but the numerical comparison between the theoretical and experimental values of $\chi / \chi_{\mathrm{P}}$ as a function of $k T / \delta$ does. The detailed analysis was done in the following way. According to the interpretation of C.E.S.R. for small silver particles [2], only particles with a diameter smaller than about $15 \AA$ contribute to the resonance signal.

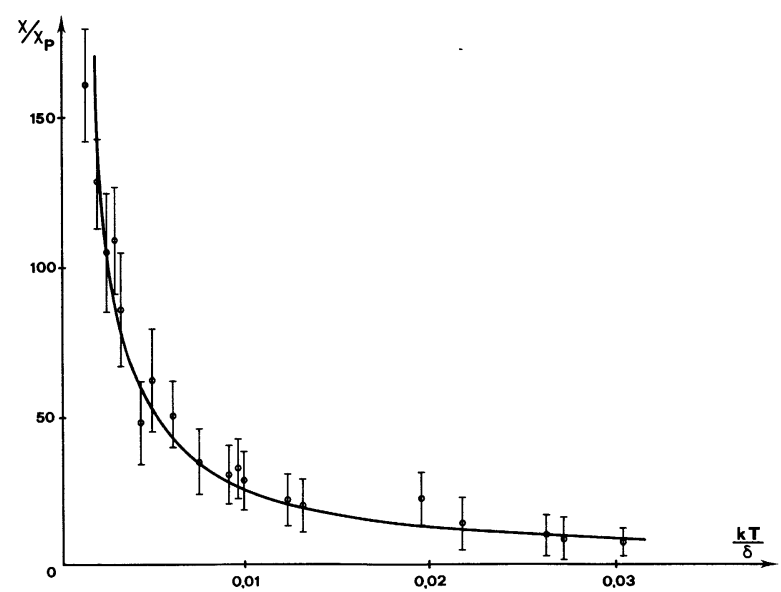

FIG. 3. - Comparison of the experimental susceptibility values of small silver particles in a benzene matrix with the theoretical behaviour of $\chi_{\text {odd }} / \chi_{\mathrm{P}}$ vs. temperature.

In a first approximation knowing the size distribution and the total amount of silver in the sample, an estimation can thus be made of the total volume $V_{0}$ of those crystals which are small enough to be observed by C.E.S.R. Together with the absolute value of the integrated signal, this value of $V_{0}$ is used to calculate the ratio $\chi / \chi_{\mathbf{p}}$. The average number of atoms $N$ necessary to calculate $\delta$ is obtained from the absolute value of $\chi$ assuming that a small odd particle behaves like one paramagnetic center of spin 1/2. This assumption is in agreement with the measured Curie temperature dependence and the fact that for particles of diameter smaller than $15 \AA$ the ratio $k T / \delta$ remains smaller than 1 at all temperatures lower than $200 \mathrm{~K}$.

It can easily be shown with the previously mentioned definition of $N$ and $\delta$, that the graph of $\chi / \chi_{\mathrm{P}}$ as a function of $k T / \delta$ is the same irrespective of the exact shape of the size distribution, and it can thus be compared with the theoretical curve calculated for particles of the same diameter. The agreement, as can be seen in figure 3 , between the theoretical and experimental curve is good. It should be noted that the agreement does not depend on the estimate of $V_{0}$ since the experimental values of $\chi / \chi_{\mathrm{P}}$ and $k T / \delta$ are respectively proportional to $1 / V_{0}$ and $V_{0}$. The $\delta$ value calculated as noted above is equal to $0.57 \mathrm{eV}$ which corresponds to small particles of approximately $7 \AA$. This number is smaller than the average from the size distribution, but the discrepancy can be understood if we take into account the fact that the real size distribution may be slighty ${ }^{-}$different from that reported in figure 2, which was obtained after evaporation of the benzene matrix, also it is possible that there are errors in the determination of $V_{0}$.

The primary result of this paper is the observation of a Curie-like susceptibility for small silver particles [12]. This result, together with the measurements of the even particles susceptibility obtained from the 
Knight shift measurements, is a confirmation of the lifting of the degeneracy as suggested by Kubo. The transition from the Curie to the Pauli behaviour could unfortunately not be observed since when we increase the temperature above $200 \mathrm{~K}$ the C.E.S.R. signal disappears. The observation of such a transition would give a definite proof that the susceptibility reported in this paper is due to small silver particles. We are planning experiments in this direction as well as double resonance experiments on small particles.

Acknowledgments. - The authors would like to thank Pr. J.-P. Borel and Pr. A. Châtelain for their backing during the course of this study, as well as for the helpful discussions they had with them. Fruitful discussions with Pr. J. Buttet and Dr. L. Boatner are gratefully acknowledged.

\section{References}

[1] Kubo, R., J. Phys. Soc. Japan 17 (1962) 975.

[2] Monot, R., Narbel, C., Borel, J.-P., Nuovo Cimento B 253 (1974).

[3] Kawabata, A., J. Phys. Soc. Japan 29 (1970) 902.

[4] Gorkov, L. P., Eliashberg, G. M., Sov. Phys. JETP 21 (1965) 940.

[5] Denton, R., Mühlschlegel, B., Scalapino, D. J., Phys. Rev. B 7 (1973) 3589.

[6] Taupin, C., J. Phys. Chem. Sol. 28 (1967) 41.

[7] Kobayashi, S., J. Phys. Soc. Japan 31 (1971) 1442.

[8] Yee, P., KnIGHt, W. D., Phys. Rev. B 11 (1975) 3261.
[9] Monot, R., Thesis, Ph. D., Ecole polytechnique fédérale de Lausanne (1975).

[10] Borel, J.-P., Borel-Narbel, C., Monot, R., Helv. Phys. Acta 47 (1974) 537.

[11] Millet, J.-L., Monot, R., 18th Ampère Congress, Nottingham (North Holland) 1974, p. 319.

[12] A recent announcement (MARzKe, R. F., Glannsinger, W. S., Bull. Am. Phys. Soc. 20 (1975) 411) stated that similar findings have been made in microcrystals of platinum. 